\title{
Approach to new diabetic mellitus patients
}

\begin{abstract}
India is the fast developing country with the population of 120 crores. The prevalence of diabetes is 17 to $23 \%$. Both urban and rural diabetic population is increasing because of deficiency in detection of prediabetic conditions. Training in diabetic management for doctors, nurses and health workers is not up to the mark. Until we fulfil all these things to concerned persons and diabetic awareness to the community it will become very difficult to prevent patients going from pre diabetic to frank Diabetes. Treating DM it is an art.
\end{abstract}

Keywords: diabetes, impaired fasting glucose, awareness, fenugreek seeds, yoga glucose, eye sight
Volume 3 Issue 6 - 2016

\section{Ramachandra K}

Consultant Diabetilogists, Srivasa Diabetic center, India

Correspondence: Ramachandra K, Consultant Diabetilogists, Srivasa Diabetic center, Behind Janatha Bazar,VV Road, Mandya 57 I 40 I, Karnataka, India, Tel +9| 9448642360, Emaildrram389@gmail.com

Received: July 17, 2016 | Published: December 02, 2016
Abbrevations: DM, diabetes mellitus; NGO, nongovernmental organisations; IFG, impaired fasting glucose; IGT, impaired glucose tolerance; RBS, random blood sugar; FBS, fasting blood sugar; PPBS, Post prandial blood sugar; PDC, pre diabetic condition

\section{Introduction}

India is the fast developing country with the population of 120 crores. Providing infrastructure for so much people is a really very big task for any government. Also, providing health care is a challenging. Diabetes is one of the most common non-communicable disease affecting 69.1 million people in India according to 2015 statistics. ${ }^{1}$ Despite numerous public health campaigns, identification of disease burden is poor because of lack of effective screening programs and adequate laboratory methods and most importantly inadequate record maintenance. The following review addresses the factors that contribute to inadequate screening programs, operational difficulties and proposes approach to new diabetic patient.

Unfortunately around $50 \%$ of people with diabetes remain undetected in India ${ }^{2}$ and many more with impaired fasting glucose (IFG) which refers to a condition in which the fasting blood glucose is elevated above what is considered normal levels but is not high enough to be classified as diabetes mellitus. It is considered a pre-diabetic state and its increasing among all sectors, both rural and urban area.

Approach to New DM is really an art, just like surgical skill. Unfortunately the trained diabetologist, physicians and general practitioners strength is very very less to treat the DM populations. We are very much lack of knowledge, information, upgrading and understanding on Diabetes. Doctors while approaching the DM patients on their first visit should exhibit great patience to hear to the patient's problems. Console them that it's not a life threatening. In authors experience this primary counselling will boost the patient's confidence and they will be receptive to new life style change which is important. Doctors with over enthusiasm should not create a sort of fear or apprehension among new DM Patients. Overt description about losing eye sight, renal problem and sexual problem etc should be avoided in initial visits. We must try our level best to give evidence based diabetic health education, awareness and try to spend minimum 20 to $30 \mathrm{~min}$ with new DM Patients.

Author will be narrating the approach to manage new DM patients which is applicable to all Asian countries (Figures 1-3): a. Screening

b. Approach

c. Summary

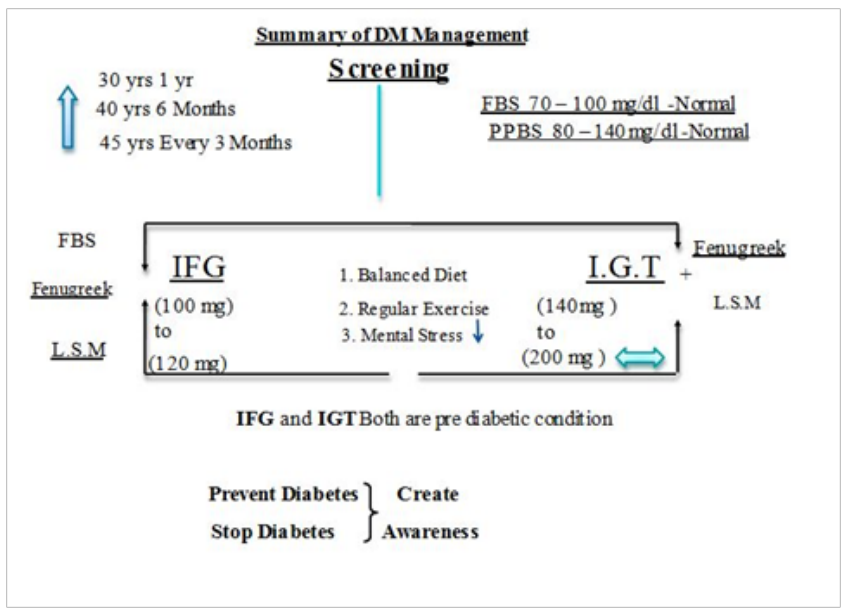

Figure I Summary of DM management of Screening.

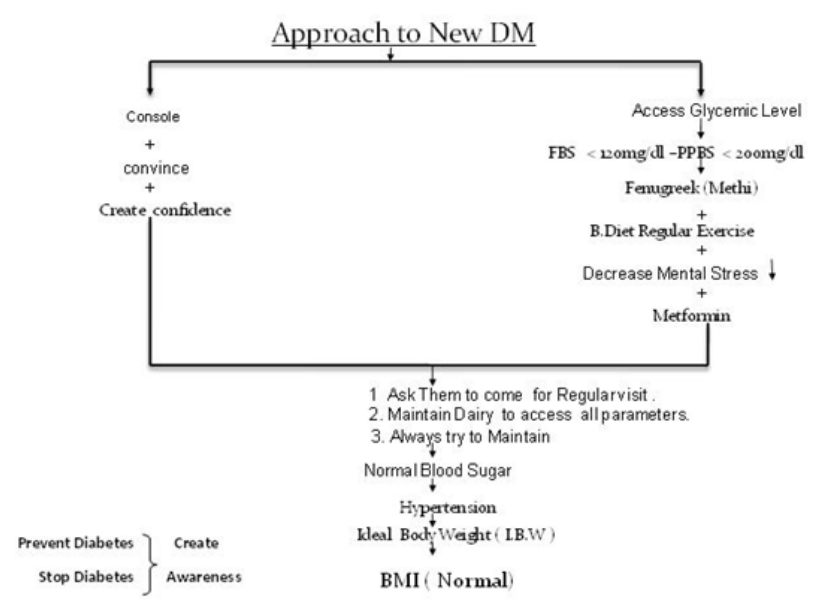

Figure 2 Approach to new DM 


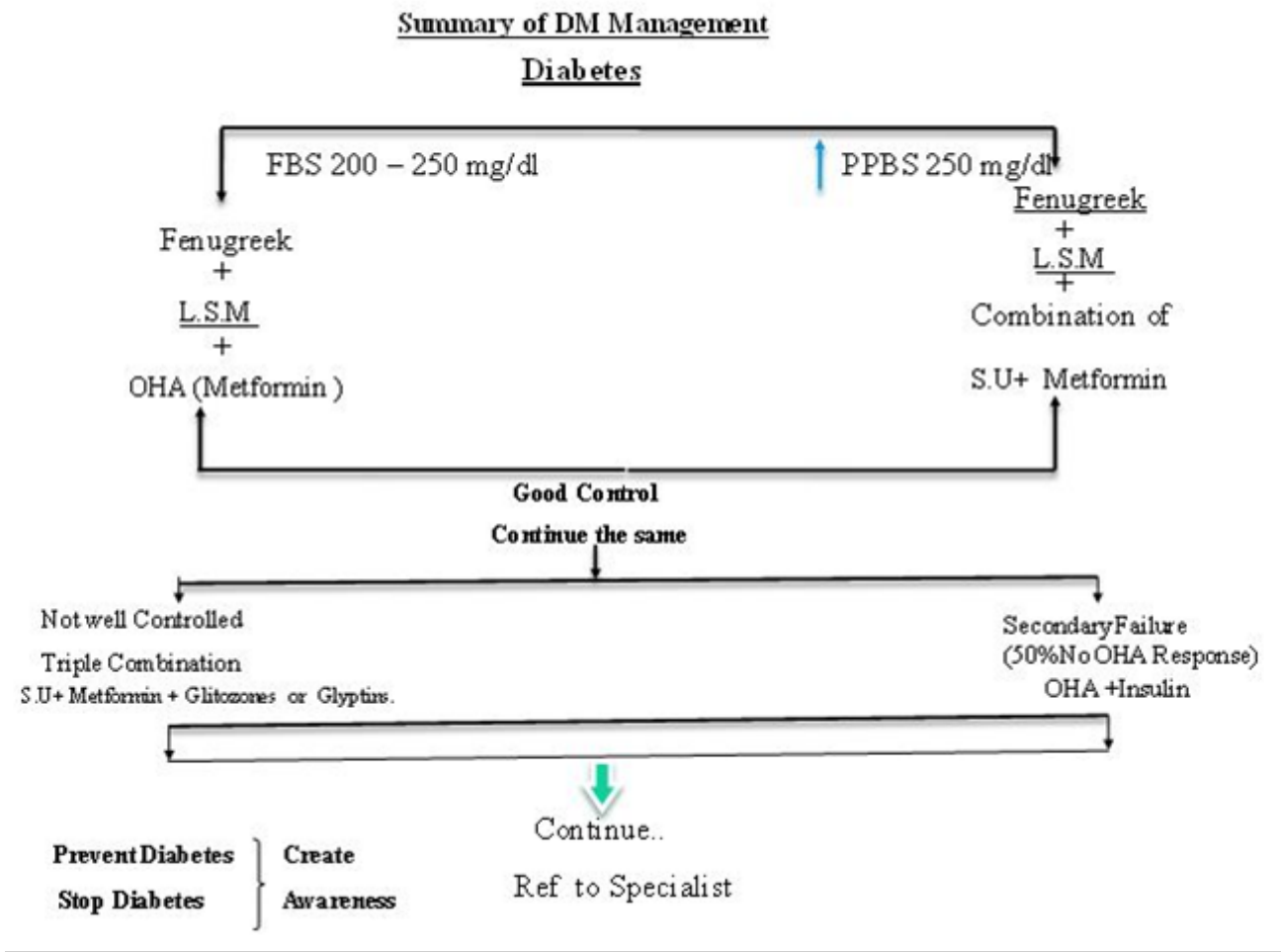

Figure 3 Summary of DM management.

\section{Screening}

The purpose of screening is to identify asymptomatic individuals who are likely to have diabetes. Annual screening for diabetes should be done all population aged more than 30yrs. Screening and early interventions are summarized in Figure 1.

\section{Approach to new patients}

Console, convince and create confidence should initial main goal in managing DM. Steps of approach is briefed in Figure 2. While managing prediabetic Fenugreek of value. Studies have shown the benefit of fenugreek seeds as an adjuvant in the control of type 2 diabetes mellitus in the form of seeds soaked in hot water. ${ }^{3,4}$ Fenu Greek powder contains safogen which act as secretagogue and $51 \%$ fibre content which delays the absorption of glucose. ${ }^{5,6}$ Preferably it should be consumed 5 to 10 minutes earlier to breakfast and dinner. In our Indian culture this is widely used in all the preparations. This will prevent prediabetic conditions going for frank diabetic condition.

\section{Summary}

Diabetes oriented training is very much required for all sectors of doctors (Diabetologist, Physicians and General Practitioners). Short term course is very much required for nurses and health workers to identify prediabetic conditions. All medical colleges should start separate diabetic department to detect and treat DM Patients. Rational way of selecting OHA'S and Medications are also very important factor provided if you monitor Balanced diet, regular exercises, life style modifications and reduce of mental stress by adopting Yoga, Dhyana and breathing exercises. Both government and politicians should play very important role for screening to detect it earlier.

\section{Remember}

Give respect-Take Respect.

Diabetes effects from head to toe.

Diabetes is not curable but learns to live with it.

Do not afraid of DM, Be happy with the existing things.

Diabetes is your life partner.

Anybody can leave you on this earth including your wife but DM won't leave you until your last breath.

\section{Acknowledgments}

None.

\section{Conflicts of interest}

The author declares there is no conflict of interest.

\section{References}

1. http://www.idf.org/membership/sea/india

2. Mohan V, Sandeep S, Deepa R, et al. Epidemiology of type 2 diabetes: Indian scenario. Indian J Med Res. 2007;125(3):217-230.

3. Neeraja A, Rajyalakshmi P. Hypoglycemic effect of processed fenugreek seeds in humans. J Food Sci Technol. 1996;33(5):427-430.

4. Raghuram TC, Sharma RD, Sivakumar B, et al. Effect of fenugreek seeds on intravenous glucose disposition in Non-insulin-dependent diabetic-patients. Phytotherapy Research. 1994;8(2):83-86. 
5. Kumar VS, Rama Rao J, Mohanty S, et al. Comparative study of fenugreek seeds on glycemic index in high and medium dietary fibre containing diets in NIDDM Patients. National Journal of Integrated Research in Medicine. 2011;2(3):29-97.
6. Neelkantan N, Naranyanan M, J de Souza R, et al. Effect of fenugreek (Trigonella foenumgraceum L.) intake of glycemia: a meta-analysis of clinical trials. Nutr J. 2014;13:7 